\title{
Measurement of Very Small Zeeman Splittings in GaN:Mn,Mg by Faraday Rotation
}

A. WoŁoś, P. Kossacki, A. Golnik, M. Kamińska, J.A. Gaj, A. TWARDOWSKI

Institute of Experimental Physics, Warsaw University

Hoża 69, 00-681 Warsaw, Poland

I. Grzegory, M. Boćkowski And S. Porowski

High Pressure Research Center, PAS, Sokołowska 29/37, 01-142 Warsaw, Poland

In this work we demonstrate an application of Faraday rotation for measuring an extremely small Zeeman splitting of an Mn related absorption line placed at $1.417 \mathrm{eV}$ in optical absorption spectrum of $\mathrm{Mn}$ and $\mathrm{Mg}$ doped gallium nitride. Analysis of the collected spectra allowed us to determine the value of the splitting as equal to $0.12 \pm 0.01 \mathrm{meV}$ at $6 \mathrm{~T}$. This data should help in establishing the nature of the observed absorption band.

PACS numbers: 78.20.Ls, 71.70.Ej, 71.55.Eq, 71.20.Nr

\section{Introduction}

Faraday rotation can be used for a precise analysis of magnetic field induced variation of sharp absorption lines, both in energy position (Zeeman splitting) and in intensity [1]. In this work we demonstrate an application of the Faraday rotation for measuring an extremely small Zeeman splitting of a sharp Mn related infrared line in optical absorption spectrum of $\mathrm{GaN}: \mathrm{Mn}, \mathrm{Mg}$, occurring in a moderate field of a few tesla.

\section{Sample and experiment}

Single GaN:Mn, Mg crystal was grown by equilibrium high pressure technique from nitrogen solution in liquid gallium. Manganese and magnesium were added 
into gallium during growth. Secondary ion mass spectroscopy (SIMS) analysis determined content of magnesium as equal to about $0.1 \%$ and content of manganese below $0.001 \%$.

Transmission and Faraday rotation were measured in a standard setup with the sample mounted strain-free in a superconducting magnet and immersed in superfluid helium. To obtain each Faraday rotation spectrum a series of transmission spectra was measured for various positions of the polarizer placed in front of the spectrograph (Fig. 1). Faraday rotation was determined by fitting parabolas (Fig. 2) to the dependence of the transmitted light intensity on the analyzer angle in the vicinity of the minimum transmission, as described in Ref. [1].

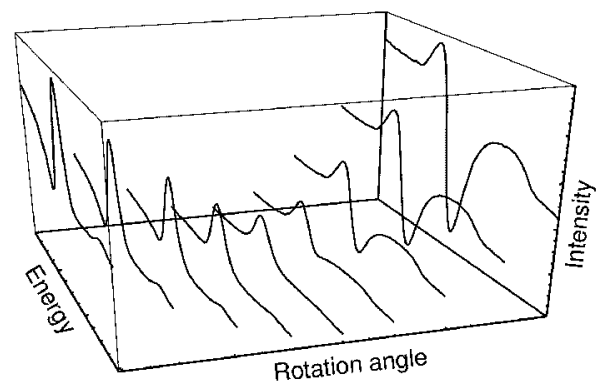

Fig. 1. Idea of Faraday rotation determination by measuring a series of transmission spectra at different positions of the analyzer.

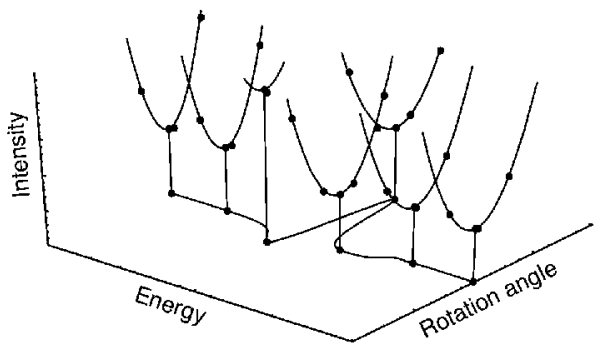

Fig. 2. Determination of the Faraday rotation spectrum by finding transmission minima vs. rotation angle.

The analysis of the spectra was based on the relation between the logarithm of the transmission and the doubled phase of the transmitted light [1], representing respectively the real and imaginary part of the same analytic function.

\section{Results and analysis}

Near infrared absorption spectrum of the sample is presented in Fig. 3. A sharp zero-phonon line (ZPL) at $1.417 \mathrm{eV}$ energy is followed by structures that 


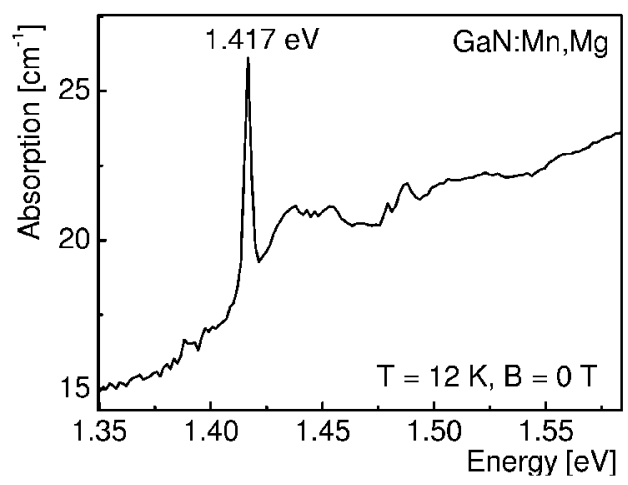

Fig. 3. Near infrared absorption spectrum of GaN:Mn,Mg.

reflect phonon density states of gallium nitride. Origin of the presented band is not fully established yet. It has been primarily attributed to photoionization transition from $\mathrm{GaN}$ valence band to bound $\mathrm{Mn}$ acceptor state [2]. Character of the band is however typical of intra-impurity transition of transition metal ions observed in GaN [3-5]. Thus an internal transition within $d$ shell of $\mathrm{Mn}$ ion cannot be excluded as the origin of the band.

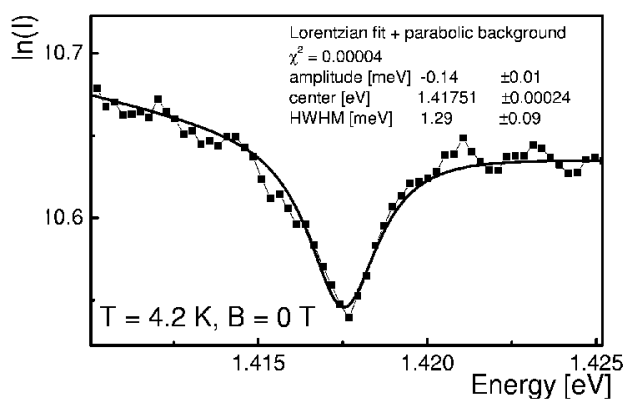

Fig. 4. Transmission in the range of zero-phonon absorption line (points) with a Lorentzian fit (solid line).

In Fig. 4 transmission spectrum of $\mathrm{GaN}: \mathrm{Mn}, \mathrm{Mg}$ in energy range of $\mathrm{ZPL}$ together with a Lorentzian fit is presented. Lorentzian fit with a parabolic background reproduces well the spectrum, according to the equation

$$
\ln (I(\omega))=A \operatorname{Re}\left(\frac{\mathrm{i}}{\omega_{0}-\omega+\mathrm{i} \gamma}\right)+C \omega^{2}+D
$$

The determined values of the amplitude $\hbar A$, resonance energy $\hbar \omega_{0}$ and HWHM width $h \gamma$ are given in the onset of Fig. 4. They were used subsequently for the analysis of the rotation spectrum, measured at $6 \mathrm{~T}$ (Fig. 5). 


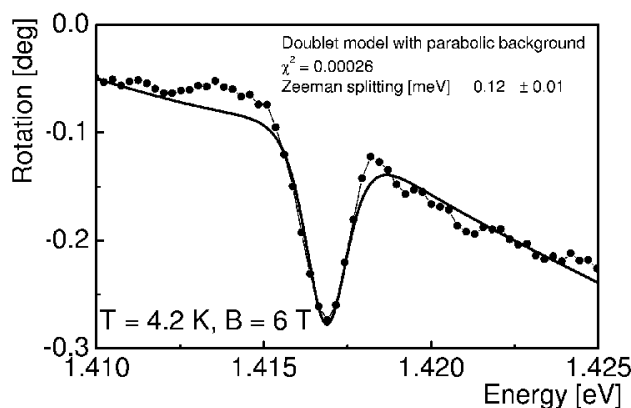

Fig. 5. Faraday rotation spectrum of the zero-phonon line. Lorentzian fit (solid) of the experimental data (points) produces Zeeman splitting value of $0.12 \mathrm{meV}$.

In principle, three possible sources of the Faraday rotation related to a given absorption line can be distinguished, namely differences between its two circularly polarized components in energy (Zeeman effect), in intensity, and/or in width. As pointed out in Ref. [1], for a symmetric line, the Zeeman mechanism produces also a symmetric rotation spectrum, whereas the two other mechanisms lead to asymmetric, dispersive type shapes. We found that the measured rotation spectra can be well described by the symmetric Zeeman type shape, therefore we exclude the other mechanisms from the interpretation. The experimental Faraday rotation spectrum was fitted with the expression

$$
\vartheta(\omega)=\frac{1}{4} \Delta \omega A \frac{\partial}{\partial \omega_{0}} \operatorname{Im}\left(\frac{\mathrm{i}}{\omega_{0}-\omega+\mathrm{i} \gamma}\right)+F \omega^{2}+G
$$

using the line parameters determined previously from the zero-field transmission spectrum (allowing a slight readjustment of $\omega_{0}$ for the shift in magnetic field). A parabolic background $F \omega^{2}+G$ was also allowed. The expression was obtained assuming a Zeeman splitting into two components of opposite circular polarizations, separated by $\Delta \omega$ much smaller than the line width. The factor $1 / 4$ results

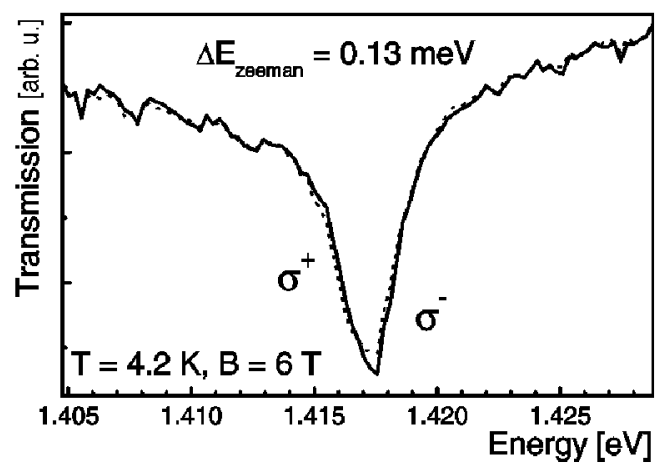

Fig. 6. Direct absorption experiment in magnetic field $6 \mathrm{~T}$ reveals Zeeman splitting of the zero-phonon line of about $0.13 \mathrm{meV}$. 
from taking into account the fact that the rotation angle is equal to half of the phase shift between the two components. A good fit was obtained, yielding Zeeman splitting value $\hbar \Delta \omega=0.12 \mathrm{meV}$, indeed much smaller than the line width. This result is consistent with direct observation of the splitting between the two circular components (Fig. 6).

\section{Conclusions}

Faraday rotation experiment combined with direct absorption measurements performed on a $\mathrm{GaN}: \mathrm{Mn}, \mathrm{Mg}$ bulk crystal allowed us to reveal a very small influence of the magnetic field on a zero-phonon line at $1.417 \mathrm{eV}$. Assuming a doublet structure of the line we obtained a value of $0.12 \pm 0.01 \mathrm{meV}$ for its Zeeman splitting at $6 \mathrm{~T}$. This data should help in establishing the nature of the observed absorption band.

\section{Acknowledgment}

This work was partially supported by the State Committee for Scientific Research (project 2P03B09418).

\section{References}

[1] W. Maślana, W. Mac, J.A. Gaj, P. Kossacki, A. Golnik, J. Cibert, S. Tatarenko, T. Wojtowicz, G. Karczewski, J. Kossut, Phys. Rev. B 63, 165318 (2001).

[2] R.Y. Korotkov, J.M. Gregie, B.W. Wessels, Appl. Phys. Lett. 80, 1731 (2002).

[3] K. Maier, M. Kunzer, U. Kaufmann, J. Schneider, B. Monemar, I. Akasaki, H. Amano, Mater. Sci. Forum 143-147, 93 (1994).

[4] J. Baur, U. Kaufmann, M. Kunzer, J. Schneider, H. Amano, I. Akasaki, T. Detchprohm, K. Hiramatsu, Mater. Sci. Forum 196-201, 55 (1995).

[5] J. Baur, K. Maier, M. Kunzer, U. Kaufmann, J. Schneider, H. Amano, I. Akasaki, T. Detchprohm, K. Hiramatsu, Appl. Phys. Lett. 64, 857 (1994). 